\title{
METHODS FOR DETERMINING THE PERIODICITY PARAMETER OF A MATHEMATICAL MODEL OF MASSIF AROUND A MINE WORKING
}

\author{
L. S. Ksendzenko ${ }^{1}$, A. S. Losev ${ }^{2} *$ \\ ${ }^{1}$ Far Eastern Federal University, 8 Sukhanova st., Vladivostok, Russian Federation \\ ${ }^{2}$ Federal State Institution of Science Institute for Applied Mathematics, Far-Eastern Branch \\ of the Russian Academy of Sciences, 7 Radio st., Vladivostok, Russian Federation
}

*Corresponding author. E-mail: A.S.Losev@yandex.ru; address for correspondence: 7, ul. Radio, Vladivostok, 690041, Russian Federation. Tel: +7 (423) 2312375

The paper presents a comparative analysis of the analytical and numerical determination of the periodicity parameter of a model of zonal rock mass destruction around a deep circular tunnel and field data from different deposits. A methodology for a simultaneous application of the analytical and numerical approaches, depending on the problems to be solved at a deposit being investigated, is substantiated.

Key words: highly compressed rock mass, zonal array destruction, non-Euclidean mathematical model parameters.

DOI: $10.17804 / 2410-9908.2016 .3 .006-013$

\section{References}

1. Guzev M.A., Paroshin A.A. Non-Euclidean Model of the Zonal Disintegration of Rocks around an Underground Working. Journal of Applied Mechanics and Technical Physics, 2001, vol. 42, iss. 1, pp. 131-139. DOI: 10.1023/A:1018877015940.

2. Qian Q.H., Zhou X.P. Non-Euclidean continuum model of the zonal disintegration of surrounding rocks around a deep circular tunnel in a non-hydrostatic pressure state. Journal of Mining Science, 2011, vol. 47, no. 1, pp. 37-46. DOI: 10.1134/S1062739147010059.

3. Tsihu T.S., Chzhu K., Ksi E. Effect of horizontal stresses on the phenomenon of zonal disintegration of rock masses with a circular tunnel. Fiziko-tekhnicheskie problemy razrabotki poleznykh iskopaemykh, 2012, no. 2, pp. 88-97. (In Russian).

4. Zhou X-P., Shou Y-D. Excavation-induced zonal disintegration of the surrounding rock around a deep circular tunnel considering unloading effect. International Journal of Rock Mechanics \& Mining Sciences, 2013, vol. 64, pp. 246-257. DOI: 10.1016/j.ijrmms.2013.08.010.

5. Zhou X., Qian Q., Song H. The effects of three-dimensional penny-shaped cracks of zonal disintegration of the surrounding rock masses around a deep circular tunnel. Acta Mechanica Solida Sinica, 2015, vol. 28, iss. 6, pp. 722-734. DOI: 10.1016/S0894-9166(16)30012-X.

6. Qian Q., Zhou X. Quantitative analysis of rockburst for surrounding rocks and zonal disintegration mechanism in deep tunnels. Journal of Rock Mechanics and Geotechnical Engineering, 2011, vol. 3, iss. 1, pp. 1-9. DOI: 10.3724/SP.J.1235.2011.00001.

7. Ksendzenko L.S. Developing a method for the determination of the parameters of zonal destruction structure of highly compressed rock mass around underground mine tunnels. Vestnik Dalnevostochnogo gosudarstvennogo tekhnicheskogo universiteta, 2011, no. 3/4 (8/9), pp. 144-166. (In Russian).

8. Ksendzenko L.S., Makarov V.V., Opanasyuk V.N., Golosov A.M. Zakonomernosti deformirovaniya i razrusheniya silno szhatykh gornykh porod $i$ massivov [Regularities in the Deformation and Destruction of Highly Compressed Rrocks and Rock Masses]. Vladivostok, DVFU Publ., 2014. 219 p. (In Russian). 
ittpi://dream-journal.or"n

9. Makarov V.V., Guzev M.A., Odintsev V.N., Ksendzenko L.S. Periodical zonal character of damage near the openings in highly-stressed rock mass conditions. Journal of Rock Mechanics and Geotechnical Engineering, 2016, vol. 8, iss. 2, pp. 164-169. DOI: 10.1016/j.jrmge.2015.09.010.

10. Li S., Wang H., Qian Q., Fan Q., Yuan L., Xue J., Zhang Q. In-situ monitoring research on zonal disintegration of surrounding rock mass in deep mine roadways. Chinese Journal of Rock Mechanics and Engineering, vol. 27, iss. 8, pp. 1545-1553. 
Подана в журнал: 11.04 .2016

УДК 622.281:539

DOI: $10.17804 / 2410-9908.2016 .3 .006-013$

\title{
СПОСОБЫ ОПРЕДЕЛЕНИЯ ПАРАМЕТРА ПЕРИОДИЧНОСТИ МАТЕМАТИЧЕСКОЙ МОДЕЛИ МАССИВА ВОКРУГ ГОРНОЙ ВЫРАБОТКИ
}

\author{
Л. С. Ксендзенко ${ }^{1}$, А. С. Лосев ${ }^{2 *}$ \\ ${ }^{1}$ Дальневосточный федеральный университет, ул. Суханова, 8., Владивосток, Российская Федерация \\ ${ }^{2}$ Федеральное государственное бюджетное учреждение науки Институт прикладной математики Дальнево- \\ сточного отделения Российской академии наук, ул. Радио, 7, Владивосток, Российская Федераиия
}

*Ответственный автор. Электронная почта: A.S.Losev@yandex.ru; адрес для переписки: ул. Радио, 7, 690041, Владивосток, Российская Федерация. Телефон: +7 (423) 231-23-75

В работе проведен сравнительный анализ аналитического и численного определения параметра периодичности модели зонального разрушения массива горных пород вокруг глубокой выработки круглого сечения с натурными данными различных месторождений. Обосновывается методика совместного использования аналитического и численного подхода в зависимости от решаемых задач на исследуемом месторождении.

Ключевые слова: сильно сжатый массив, зональное разрушение массива, параметры неевклидовой математической модели.

\section{1. Введение}

На сегодняшний день исследования горных пород являются приоритетными направлениями во многих странах в решении задач, связанных с горным делом, задействованы специалисты различного уровня всего мира. В процессе поиска новых методов и подходов в этой научной области один из значимых результатов был получен М.А. Гузевым и А.А. Парошиным. Ими впервые была предложена неевклидова модель сплошной среды для описания распределения поля напряжений вокруг выработки круглого сечения [1]. Отказавшись от условия совместности деформаций Сен-Венана, для случая плоской деформации, авторы ввели функцию дефектности массива горной породы, удовлетворяющую бигармоническому уравнению с заданными граничными условиями. Полученный результат стал основой новых исследований и позволил по-новому взглянуть на явления зонального разрушения массива горных пород вокруг глубоких подземных выработок [2-8].

В частности, авторами работы [2] решена краевая задача о распределении поля напряжений вокруг выработки в случае плоской деформации и в условиях негидростатического нагружения. Задача представлена в виде двух составляющих, за счет разложения поля упругих напряжений в сумму полей: первое из них вызвано несовместными деформациями нарушенных зон, а второе - совместными деформациями ненарушенных зон. В первом случае упругие напряжения определяются с помощью неевклидовой модели, во втором - с помощью классической упруго механической модели. В итоге, с использованием силового критерия Кулона-Мора, установлено количество нарушенных зон, их местоположение, которые зависят от физико-механических свойств материала породы, а также от его неевклидовых параметров.

В работе [3] на основе представленной неевклидовой модели зональной дезинтеграции массива горных пород вокруг выработки круглого сечения, определено количество и размер нарушенных и ненарушенных зон. Показана зависимость количества и размера зон от продольного натурного напряжения, тангенциального и радиального напряжений, коэффициента промежуточного главного напряжения, а также классификационного коэффициента RMR (Rock Mass Rating).

Ksendzenko L.S. et al. / Methods for determining the periodicity parameter of a mathematical model of massif around a mine working 
В работе [4] предложена динамическая модель исследования явлений зональной дезинтеграции во вмещающем массиве горных пород вокруг глубоких круглых туннелей, подвергнутых динамической разгрузке в условиях гидростатического сжатия. На основе неравновесной термодинамики получено неевклидово динамическое уравнение, решение которого определяется прямыми и обратными преобразованиями Лапласа. Показано, что число нарушенных зон возрастает с увеличением гравитационного напряжения, параметра нарушенности горной породы (disturbance factor), скорости разгрузки, а также с уменьшением предела прочности на одноосное сжатие, геологического индекса прочности (GSI), параметра прочности ті и коэффициента Пуассона $v$.

В работе [5] на основе новой неевклидовой модели исследовано влияние на зональную дезинтеграцию горных пород глубоких выработок пенни-образных трещин и естественного осевого напряжения. Применен коэффициент плотности энергии деформации при определении коэффициента интенсивности напряжений в вершинах пенни-образных трещин. Путем численных расчетов установлено, что величина и расположение зон разрыва чувствительны к микро- и макро-механическим параметрам, а также к величине напряжения в нетронутом массиве.

В работе [6] на основе неевклидовой модели изучается связь между зональной дезинтеграцией и горными ударами. Исследуется механизм зарождения вторичных микротрещин, механизм их перехода в неустойчивое состояние, распространение и объединение вторичных микротрещин, образование макротрещин (что ведет к горному удару). ниям:

Не смотря на уникальность работ все авторы [2-6] приходят к следующим заключе-

- в условиях больших глубин закономерным является зональный характер разрушения массива горных пород вокруг подземных выработок;

- свойствами структур зонального разрушения являются повторение контура выработки и чередование разрушенных и относительно ненарушенных горных пород;

- неевклидовы математические модели, наиболее адекватно описывают явление зональной дезинтеграции.

Общим существенным пробелом в рассмотренных работах является отсутствие сравнения с натурными экспериментами и частичная проработка алгоритма определения параметров построенных математических моделей. Поэтому, актуальным остается вопрос качества определения параметров построенных моделей, а также влияние точности их вычисления на исследуемые физические эффекты и горные явления.

\section{2. Распределение поля напряжений вокруг выработки круглого сечения}

Обратимся к задаче о распределении поля напряжений вокруг выработки круглого сечения, которая рассматривается как плоская и стационарная, в условиях несжимаемости и гидростатичности нагружения на бесконечности [1]. Задача решается на основе полученного уравнения равновесия

$$
\frac{\partial \sigma_{\mathrm{rr}}}{\partial \mathrm{r}}+\frac{1}{\mathrm{r}}\left(\sigma_{\mathrm{rr}}-\sigma_{\varphi \varphi}\right)=0
$$

бигармонического уравнения для функции дефектности

$$
\Delta^{2} R-\gamma^{2} R=0
$$

и граничных условий 


$$
\left.R\right|_{r=r_{0}}=0,\left.\frac{\partial R}{\partial r}\right|_{r=r 0}=0,
$$

где $\sigma_{\text {тr }}$ - нормальное радиальное напряжение; $\sigma_{\varphi \varphi}$ - нормальное тангенциальное напряжение; $\sigma_{\mathrm{r} \varphi}-$ касательное напряжение; $\Delta$ - оператор Лапласа; $\gamma$ - параметр периодичности модели. В полярных координатах для бигармонического уравнения

$$
\left(\frac{\partial^{2}}{\partial r^{2}}+\frac{1}{r} \frac{\partial}{\partial r}\right)^{2} R=\gamma^{2} R
$$

определено решение при условии $r \rightarrow \infty$ для расстояния от центра выработки до точки массива в виде равенства:

$$
R(r)=a J_{0}(\sqrt{\gamma} r)+b N_{0}(\sqrt{\gamma} r)+c K_{0}(\sqrt{\gamma} r)
$$

где $J_{0}, N_{0}, K_{0}$ - функции Бесселя, Неймана и Макдональда нулевого порядка.

В [7] решение бигармонического уравнения (2), в отличие от [1], найдено для граничных условий:

$$
\left.\frac{\partial R}{\partial r}\right|_{r=r_{0}}=0,\left.\frac{\partial R}{\partial r}\right|_{r=r^{*}}=0
$$

соответствующих зональному характеру разрушения массива во всех зонах по принципу: первое граничное условие для функции $R$ определяем как ее экстремальность на контуре выработки, а второе - как экстремальность в середине первой зоны разрушения и т.д.

В данной постановке задачи и полученном решении актуальным остается вопрос качественного и точного определения параметров рассматриваемой модели. Особенно важным моментом является степень точности определения параметра периодичности и его влияние на точность прогнозов, числа, положения и протяженности зон разрушения массива горных пород. В ходе рассмотрения данного вопроса было разработано два независимых подхода определения параметра $\gamma$.

\section{3. Методика и результаты определения параметра периодичности модели зонального разрушения массива горных пород}

Аналитический подход. На основе натурных экспериментов для исследованных месторождений была получена аналитическая зависимость между параметром $\gamma$ и расстоянием от контура выработки до середины первой зоны разрушения в единицах радиуса выработки

$$
\gamma\left(r * / r_{0}\right)=-10\left(r * / r_{0}\right)+23
$$

где $r^{*}$ - середина первой зоны разрушения, считая от контура выработки; $r_{0}$ - радиус выработки, м [7].

Численный подход. Способ основан на численном подборе параметра $\gamma$, при котором параметры $a$ и $b$ функции дефектности принимают наибольшие отрицательные значения на контуре выработки, что характеризуется достижением функция дефектности на контуре выработки экстремального значения и соответственно наличием зоны разрушения [8]. 
Каждый из предложенных подходов обладает своими достоинствами и недостатками, в частности, аналитический подход определяет параметр $\gamma$ с точностью до целых, не до конца апробирован на неизвестных месторождениях, но является очень доступным и простым в плане вычисления. Численный подход является общим способом определения параметра $\gamma$, позволяющим вычислить искомый параметр с заданным уровнем точности, но требует весьма трудоемких вычислений.

В ходе расчета параметра периодичности для исследованных месторождений по обеим методикам было получено, что расхождение в точности в некоторых случаях доходит до $11,21 \%$.

При детальном рассмотрении для Норильского месторождения и угольной шахты Dingji (КНР) [10] было установлено, что в сравнении с натурными данными чувствительность построенной модели по отношению к параметру $\gamma$ определяется двумя знаками после запятой. После чего для рассматриваемых месторождений был проведен сравнительный анализ натурных [10] и экспериментальных данных с численным и аналитическим подходом.

Таблица 1 - Норильское месторождение

\begin{tabular}{|c|c|c|c|c|}
\hline \multirow{2}{*}{$\begin{array}{c}\text { Значение } \\
\text { параметра } \gamma\end{array}$} & \multicolumn{3}{|c|}{$\begin{array}{c}\text { Относительная погрешность вычисления ближней } \\
\text { и дальней границ зон разрушения, \% }\end{array}$} \\
\cline { 2 - 5 } & \multicolumn{2}{|c|}{ I зона } & \multicolumn{2}{c|}{ II зона } \\
\hline$\gamma=3$ & 0,71 & 4,86 & 19,06 & 6,8 \\
\hline$\gamma=3,2$ & 2,14 & 5,36 & 17,18 & 5,29 \\
\hline$\gamma=3,29$ & 3,57 & 5,67 & 16,25 & 4,53 \\
\hline
\end{tabular}

Таблица 2 - Угольная шахта Dingji (KHP)

\begin{tabular}{|c|c|c|c|c|c|c|c|c|}
\hline \multirow{2}{*}{$\begin{array}{c}\text { Значение пара- } \\
\text { метра } \gamma\end{array}$} & \multicolumn{6}{|c|}{$\begin{array}{r}\text { Относительная погрешность вычисления ближней } \\
\text { и дальней границ зон разрушения, \% }\end{array}$} \\
\cline { 2 - 10 } & \multicolumn{2}{|c|}{ I зона } & \multicolumn{2}{|c|}{ II зона } & \multicolumn{2}{c|}{ III зона } & \multicolumn{2}{|c|}{ IV зона } \\
\hline$\gamma=9$ & 0 & 2,69 & 10,6 & 10,8 & 11,6 & 3,00 & 9,51 & 7,04 \\
\hline$\gamma=9,9$ & 0 & 1,81 & 8,09 & 8,17 & 8,00 & 0,13 & 5,45 & 3,44 \\
\hline$\gamma=9,93$ & 0 & 1,61 & 8 & 8,07 & 7,88 & 0,22 & 5,33 & 3,33 \\
\hline
\end{tabular}

Расчет относительной погрешности для рассматриваемых месторождений показывает, что увеличение числа знаков после запятой положительно отражается на вычислении границ зон разрушения в общем случае (табл. 1-2). В частности, для Норильского месторождения можно заметить, что относительная погрешность вычисления границ первой зоны разрушения меньше в случае, когда $\gamma=3$, т.е. параметр принимает целочисленное значение (табл. 1). По Угольной шахте Dingji (KHP) анализ результатов показывает, что увеличение числа знаков после запятой в параметре $\gamma$ понижает погрешность счета для вычисления границ всех зон разрушения (табл. 2). При более детальном рассмотрении можно сказать, что для первой зоны увеличение числа знаков носит уточняющий характер, так как погрешность уменьшается не более чем на $1 \%$, в то время как относительная погрешность остальных зон разрушения сокращается намного сильнее на $2-4 \%$. 


\section{4. Заключение}

Таким образом, нельзя окончательно утверждать, какой из подходов вычисления параметра периодичности лучше. Поэтому в зависимости от поставленной задачи предлагается использовать оба подхода численный и аналитический. А именно - при первичном анализе выработки на новом месторождении, когда необходимо решить вопрос дальнейшей разработки выработки в данном месте, целесообразно использовать аналитический подход. Однако в случае более детального исследования, требующего определения числа зон, их радиальной протяженности, глубины появления зоны, точное положение последней зоны разрешения как границы области влияния одиночной выработки, конечно, требуются более точные данные, которые возможно получить численным методом.

В результате согласованного использования численного и аналитического способа определения параметра периодичности модели зонального разрушения массива горной породы достигается более качественная сходимость теоретических результатов с натурными. Данное согласование позволяет понизить ошибку относительной погрешности на этапе расчета параметров исследуемой модели, что существенно повышает точность и качество результатов полученных с её помощью в дальнейшей работе.

\section{Благодарность}

Работа выполнена при поддержке Российского научного фонда № 14-11-00079.

\section{Литература}

1. Guzev M. A., Paroshin A. A. Non-Euclidean Model of the Zonal Disintegration of Rocks around an Underground Working // Journal of Applied Mechanics and Technical Physics. - 2001. Vol. 42, iss. 1. - P. 131-139. - DOI: 10.1023/A:1018877015940.

2. Qian Q., Zhou X. Non-Euclidean continuum model of the zonal disintegration of surrounding rocks around a deep circular tunnel in a non-hydrostatic pressure state // Journal of Mining Science. - 2011. - Vol. 47, no. 1. - P. 37-46. - DOI: 10.1134/S1062739147010059.

3. Циху Ц., Чжу К., Кси Е. Влияние горизонтальных напряжений на явление зональной дезинтеграции горных пород в массиве с выработкой круглого сечения // ФТПРПИ. - 2012. № 2. - C. 88-97.

4. Zhou X-P., Shou Y-D. Excavation induced zonal disintegration of the surrounding rock around a deep circular tunnel considering unloading effect // International Journal of Rock Mechanics \& Mining Sciences. - 2013. - Vol. 64. - P. 246-257. - DOI: 10.1016/j.ijrmms.2013.08.010.

5. Zhou X., Song H., Qian Q. The effects of three-dimensional penny-shaped cracks of zonal disintegration of the surrounding rock masses around a deep circular tunnel // Acta Mechanica Solida Sinica. - 2015. - Vol. 28, iss. 6. - P. 722-734. - DOI: 10.1016/S0894-9166(16)30012-X.

6. Qian Q., Zhou X. Quantitative analysis of rockburst for surrounding rocks and zonal disintegration mechanism in deep tunnels // Journal of Rock Mechanics and Geotechnical Engineering. 2011. - Vol. 3, iss. 1. - P. 1-9. - DOI: 10.3724/SP.J.1235.2011.00001.

7. Ксендзенко Л. С. Разработка метода определения параметров зональной структуры разрушения сильно сжатого массива вокруг подземных выработок // Вестник Дальневосточного государственного технического университета. - 2011. - № 3/4 (8/9). - С. 144-166.

8. Закономерности деформирования и разрушения сильно сжатых горных пород и массивов: монография / Л. С. Ксендзенко, В. В. Макаров, В. Н. Опанасюк, А. М. Голосов. Владивосток : ДВФУ, 2014. - 219 с.

9. Periodical zonal character of damage near the openings in highly-stressed rock mass conditions / V. V. Makarov, M. A. Guzev, V. N. Odintsev, L. S. Ksendzenko // Journal of Rock Mechanics and Geotechnical Engineering. - 2016. - Vol. 8, iss. 2. - P. 164-169. DOI: 10.1016/j.jrmge.2015.09.010. 
10. In-situ monitoring of zonal disintegration of surrounding rock mass in deep mine roadways / S. Li , H. Wang, Q. Qian, Q. Fan, L. Yuan, J. Xue, Q. Zhang // Chinese Journal of Rock Mechanics and Engineering. - 2008. - Vol. 27, iss. 8. - P. 1545-1553. DOI: $10.1155 / 2015 / 230126$. 